\title{
Bott-Samelson Varieties, Subword Complexes and Brick Polytopes
}

\author{
Laura Escobart|k
}

Cornell University, Ithaca, NY, USA

\begin{abstract}
Bott-Samelson varieties factor the flag variety $G / B$ into a product of $\mathbb{C P}^{1}$ 's with a map into $G / B$. These varieties are mostly studied in the case in which the map into $G / B$ is birational; however in this paper we study fibers of this map when it is not birational. We will see that in some cases this fiber is a toric variety. In order to do so we use the moment map of a Bott-Samelson variety to translate this problem into a purely combinatorial one in terms of a subword complex. These simplicial complexes, defined by Knutson and Miller, encode a lot of information about reduced words in a Coxeter system. Pilaud and Stump realized certain subword complexes as the dual to the boundary of a polytope which generalizes the brick polytope defined by Pilaud and Santos. For a nice family of words, the brick polytope is the generalized associahedron realized by Hohlweg and Lange. These stories connect in a nice way: the moment polytope of a fiber of the Bott-Samelson map is the Brick polytope. In particular, we give a nice description of the toric variety of the associahedron.

Résumé. Les variétés de Bott-Samelson facteur le variété de drapeaux $G / B$ dans un produit de $\mathbb{C P} s^{\prime 1}$ avec une application dans $G / B$. Ces variétés sont principalement étudiés dans le cas où la application dans $G / B$ est birationnelle. Dans cet article, nous étudions les fibres de cette application quand il n'est pas birationnelle. Nous verrons que dans certains cas, cette fibre est une variété torique. Pour ce faire, nous utilisons l'application moment d'une variété BottSamelson pour traduire ce problème dans une question purement combinatoire en termes d'un complexe de sous-mot. Ces complexes simpliciaux, définies par Knutson et Miller, codent pour un grand nombre d'informations sur les mots reduits dans un groupe de Coxeter. Pilaud et Stump décrits certains complexes de sous-mots comme le dual d?une frontier d'un polytope qui généralise le polytope de briques défini par Pilaud et Santos. Pour une belle famille de mots, le polytope de briques est l?associaèdre généralisée réalisé par Hohlweg et Lange. Ces histoires se connecter d'une manière agréable: le polytope moment d'une fibre de 1?application Bott-Samelson est le polytope de briques. En particulier, nous donnons une belle description de la variété torique du associaèdre.
\end{abstract}

Keywords: Bott-Samelson variety, Subword complex, Brick polytope, Moment polytope, Toric variety, Associahedra

\section{Introduction}

The Bott-Samelson varieties were first defined in Bott and Samelson (1955). In Bott and Samelson (1961) the authors gave a desingularization of Schubert varieties in $G / B$ using these varieties. Demazure used

*Email: le78@cornell.edu.

1365-8050 (c) 2014 Discrete Mathematics and Theoretical Computer Science (DMTCS), Nancy, France 
them to analyze $H^{\circ}(G / B, \mathbb{C})$ and the projective coordinate ring $\mathbb{C}[G / B]$. Intuitively, Bott-Samelson varieties factor $G / B$ into a product of $\mathbb{C P}^{1}$ 's via a map into $G / B$. These varieties are mostly studied in the case in which the map into $G / B$ is birational.

In this paper we will study the fibers of this map when it is not birational. We will show that in some cases this fiber is a toric variety. In order to do so we will translate this problem into a purely combinatorial one in terms of subword complexes. These simplicial complexes $\Delta(Q, w)$ are parametrized by a word $Q$ in the generators of the Weyl group $W$ of $G$ and an element $w \in W$. They were defined by Allen Knutson and Ezra Miller in Knutson and Miller (2004) to describe the geometry of determinantal ideals and Schubert polynomials. In Pilaud and Stump (2011), the authors define the brick polytope and realize certain subword complexes as the boundary of a polytope dual to the brick polytope. In Ceballos et al. (2011) Ceballos, Labbé and Stump show that for a nice family of words, the brick polytope is the cluster polytope and for the Weyl group of type A it is a associahedron. In Theorem 4.4 we prove that for the words Pilaud and Stump call realizable, a fiber of the Bott-Samelson map is the toric variety of the Brick polytope. We then get a great description of the toric variety of the associahedron in terms of flags arranged in a poset.

Actually the toric case is just a shadow of a more general situation. We will see in Theorem 4.3 that for any word $Q$ and element $w \in W$ the brick polytope is the moment polytope of a fiber of the BottSamelson variety. This motivates us to define the Brick manifold as the fiber we study in this paper. This paper shows a very nice connection between three combinatorial objects and solves the toric variety case for Brick manifolds. However, there is much structure here that can be discovered.

\section{Some Definitions}

\subsection{Subword complexes}

Let $W$ be the Weyl group of a complex Lie group, i.e., $W$ is a crystallographic Coxeter group and let $S=\left\{s_{i}: i \in I\right\}$ denote its generators.

Notation: Let $Q=\left(q_{1}, \ldots, q_{k}\right)$ be a word in $S$ and let $P=\left(q_{i_{1}}, \ldots, q_{i_{k}}\right)$ be a subword of $Q$, we denote $\prod^{b} P:=\prod_{j=1}^{b} q_{i_{j}}$ and $\prod P:=\prod_{j=1}^{k} q_{i_{j}}$.

Definition 2.1 Let $Q=\left(q_{1}, \ldots, q_{k}\right)$ be a word in $S$ and $w \in W$. The subword complex $\Delta(Q, w)$ is the simplicial complex whose facets are the subwords $F$ of $Q$ such that the product $\prod Q \backslash F$ is a reduced expression for $w$. The cofacets of $\Delta(Q, w)$ are the complements of the facets.

Definition 2.2 We define the Demazure product of a word Q inductively as follows:

- $\operatorname{Dem}($ empty $w o r d)=i d$

- $\operatorname{Dem}(Q \cup s)= \begin{cases}\operatorname{Dem}(Q) \cdot s & \text { if } \ell(\operatorname{Dem}(Q) s)>\ell(\operatorname{Dem}(Q)) \\ \operatorname{Dem}(Q) & \text { if } \ell(\operatorname{Dem}(Q) s)<\ell(\operatorname{Dem}(Q))\end{cases}$

In Knutson and Miller (2004) the authors prove that $\Delta(Q, w)$ is a sphere if and only if $\operatorname{Dem}(Q)=w$; in this paper we only consider such pairs. 


\subsection{Brick polytopes}

Let $\Delta(W):=\left\{\alpha_{s}: s \in S\right\}$ be the simple roots of $W$ and let $\nabla(W):=\left\{\lambda_{s}: s \in S\right\}$ be its fundamental weights. Given a subword complex $\Delta(Q, w)$ with $|Q|=m$ define the root function $\mathrm{r}(I, \cdot)$ : $\{$ subwords of $Q\} \rightarrow \Delta(W)$ and the weight function $\mathrm{w}(I, \cdot):\{$ subwords of $Q\} \rightarrow \nabla(W)$ by

$$
\mathrm{r}(I, k):=\left(\prod^{k-1} Q \backslash I\right)\left(\alpha_{q_{k}}\right) \quad \text { and } \quad \mathrm{w}(I, k):=\left(\prod^{k-1} Q \backslash I\right)\left(\lambda_{q_{k}}\right) .
$$

Definition 2.3 The brick vector of a face $F$ of $\Delta(Q, w)$ is defined by

$$
B(F)=\sum_{k \in[m]} \mathrm{w}(F, k),
$$

and the brick polytope is the convex hull of the brick vectors of some faces of $\Delta(Q, w)$

$$
B(Q, w):=\operatorname{conv}\left\{B(F): F \in \Delta(Q, w) \text { and } \prod Q \backslash F=w\right\} .
$$

The following definition is referred to as realizing or root independent in Pilaud and Stump (2011) We will call it toric due to one of the main theorems of this paper which states that the brick manifold of a word $Q$ is toric if and only $Q$ is root independent.

Definition 2.4 $A$ word $Q$ is toric if for some vertex $B(F)$ of $B(Q, w)$ (or all vertices) we have that the multiset $r(F):=\{\{\mathrm{r}(F, i): i \in F\}\}$ is linearly independent.

\section{Brick Manifolds for $S L_{n}(\mathbb{C})$}

We start with the case $G=S L_{n}(\mathbb{C})$ both because it's nice combinatorial pictures and as a motivation to the general complex semi simple Lie group case.

\subsection{Definition of Bott-Samelson varieties for $S L_{n}(\mathbb{C})$}

Let $G=S L_{n}(\mathbb{C})$ and fix an ordered basis for $\mathbb{C}^{n}$. Let $B$ be the subgroup $S L_{n}(\mathbb{C})$ consisting of upper triangular matrices with respect to this basis. We then get an ascending flag of vector spaces

$$
\left\langle e_{1}\right\rangle \subset \cdots \subset\left\langle e_{1}, \ldots, e_{n}\right\rangle .
$$

Let $T$ be the maximal torus contained in $B$, then $T$ consists of all diagonal matrices. The matrices in the minimal parabolic subgroups $P_{i}$ are almost upper triangular, except they have a possibly nonzero entry at the position $(i+1, i)$. The quotient $G / B$ is the flag variety, that is, the space of flags $\{0\} \subset V_{1} \subset \cdots \subset$ $V_{n}=\mathbb{C}^{n}$ where each $V_{i}$ is an $i$-dimensional vector space. Moreover, the Weyl group of $G$ is $W=A_{n-1}$.

We begin the definition of $B S^{Q}$ by an example

Example 3.1 Let $G=S L_{3}(\mathbb{C})$ and $Q=\left(s_{1}, s_{2}, s_{1}, s_{2}, s_{1}\right)$, then the Bott-Samelson variety $B S^{Q}$ is constructed iteratively by reading the word from left to right: if $k$-th letter of $Q$ is $s_{i}$, we have an $i$-th dimensional vector space $V_{k}$ such that $V_{k-1} \subset V_{k} \subset V_{k+1}$. In this example we have that

$$
B S^{Q}=\left\{\left(V_{1}, V_{2}, V_{3}, V_{4}, V_{5}\right) \text { : each ascending chain in the poset below is a complete flag }\right\}
$$




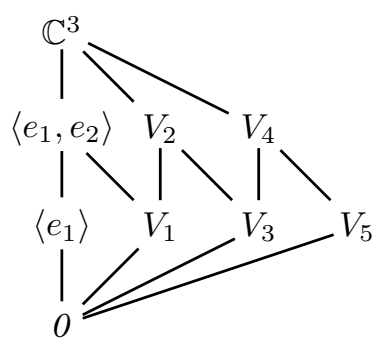

More generally, if $Q=\left(q_{1}, \ldots, q_{m}\right)$ then $B S^{Q}$ is a list of $m+1$ flags where the first one is the base flag and such that each one agrees with the previous one except possibly in one subspace. We can give a point in $B S^{Q}$ by giving subspaces $\left(V_{1}, \ldots, V_{m}\right)$ such that the incidence relations given by the flags hold. The map $B S^{Q} \stackrel{\mathrm{m}_{\mathrm{Q}}}{\longrightarrow} G / B$ maps the list to the last flag.

Example 3.2 Continuing with the previous example, we have that $m_{Q}: B S^{\left(s_{1}, s_{2}, s_{1}, s_{2}, s_{1}\right)} \rightarrow G / B$ is the map

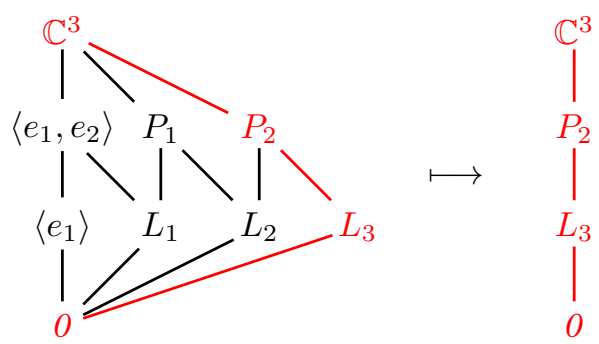

Note that $T$ acts on $\mathbb{C}^{n}$ by multiplication, and this action can be extended to $T$ acting on $B S^{Q}$. In Section 4.1, we will show that Bott-Samelson varieties are Hamiltonian symplectic manifolds with respect to this torus action. Therefore, they have moment maps with respect to the torus action

$$
\mu: B S^{Q} \longrightarrow \mathbb{R}\langle\nabla(W)\rangle,
$$

where $\mathbb{R}\langle\nabla(W)\rangle$ is real span of the fundamental weights of $W$. We now describe this moment map very concretely. Given $p=\left(V_{1}, \ldots, V_{m}\right) \in B S^{Q}$ and $i \in[m]$ define

$$
\mu(p, i)=\left(\operatorname{dim}_{e_{1}}\left(V_{i}\right), \ldots, \operatorname{dim}_{e_{m}}\left(V_{i}\right)\right),
$$

where $\operatorname{dim}_{e_{j}}(V)$ denotes the dimension of $V$ on the $e_{j}$-th coordinate, then the moment map is

$$
\begin{gathered}
B S^{Q} \stackrel{\mu}{\longmapsto} \mathbb{R}^{n} \\
\left(V_{1}, \ldots, V_{m}\right) \stackrel{\mu}{\longmapsto}\left(\sum_{i=1}^{m} \operatorname{dim}_{e_{1}}\left(V_{i}\right), \ldots, \sum_{i=1}^{m} \operatorname{dim}_{e_{n}}\left(V_{i}\right)\right) .
\end{gathered}
$$

The image of this map is the moment polytope and it equals the convex hull of the images of the $T$-fixed points. There is a 1-1 correspondence between $T$-fixed points on $B S^{Q}$ and subwords $R$ of $Q$ : if $p(R)$ is 
the $T$-fixed point corresponding to $R$ then $m_{Q}(p(R))=\left(\prod Q \backslash R\right) B \in G / B$. In more combinatorial terms, the point $p(R)$ corresponding to the subword $S$ is determined by deciding between $=$ and $\neq$ in each diamond

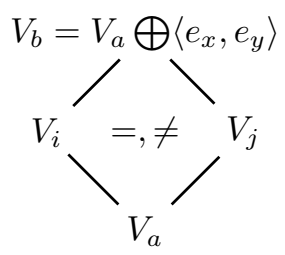

For $Q=\left(q_{1}, \ldots, q_{m}\right)$, we pick $=$ if $q_{j} \in R$ and $\neq$ if $q_{j} \notin R$. We illustrate the correspondence by an example.

Example 3.3 The subword $J=\left(-, s_{2},-,-, s_{1}\right)$ of $Q=\left(s_{1}, s_{2}, s_{1}, s_{2}, s_{1}\right)$ corresponds to the flags below and the image of $m_{Q}: B S^{Q} \rightarrow G / B$ is $\left(s_{1} s_{1} s_{2}\right) B=\left(s_{2}\right) B$.

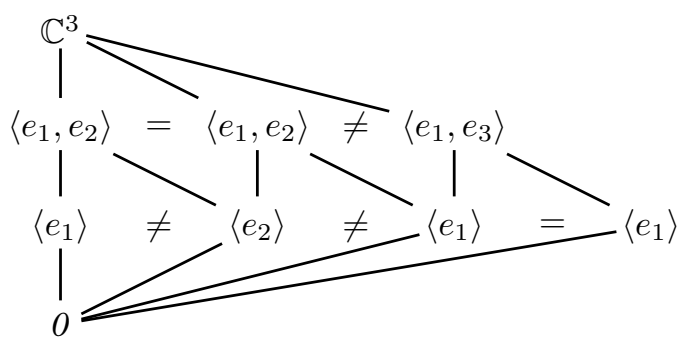

This correspondence motivates the relation between fibers of the map $m_{Q}: B S^{Q} \longrightarrow G / B$ and subword complexes.

\subsection{Brick polytopes in the $S L_{n}(\mathbb{C})$ case}

The sorting network $\mathcal{N}_{Q}$ of a word $Q=\left(q_{1}, \ldots, q_{m}\right)$ consists of $n$ horizontal lines (called the levels) and $m$ vertical segments (called the commutators) drawn from left to right so that each commutator joins consecutive levels, no two commutators share a common endpoint, and if $q_{k}=s_{i}$ then the $k$-th commutator connects levels $i$ and $i+1$. A brick of $\mathcal{N}_{Q}$ is a connected component of its complement, bounded on the right by a commutator.

A pseudoline supported by $\mathcal{N}_{Q}$ is a path on $\mathcal{N}_{Q}$ traveling monotonically from left to right. A commutator of $\mathcal{N}_{Q}$ is called a crossing between two pseudolines if it is crossed by the two pseudolines and it is called a contact if its endpoints are one on each pseudoline. A pseudoline arrangement on $\mathcal{N}_{Q}$ is a collection of $n$ pseudolines such that each two have at most one crossing and no other intersection.

Example 3.4 Let $Q=\left(s_{1}, s_{2}, s_{1}, s_{2}, s_{1}\right)$ then $w_{0}=\operatorname{Dem}(Q)=s_{1} s_{2} s_{1}=s_{2} s_{1} s_{2}$ and we have that the sorting network $\mathcal{N}_{Q}$ is

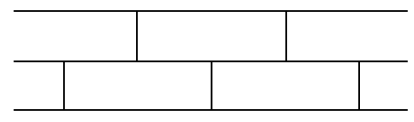


and the pseudoline arrangement corresponding to the subword $J=\left(s_{1},-,-,-, s_{1}\right)$ is

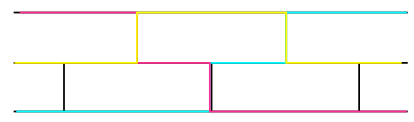

Given a pseudoline arrangement supported by $\mathcal{N}_{Q}$ with contacts $F=\left(q_{i_{1}}, \ldots, q_{i_{k}}\right)$ the product $w=$ $\prod Q \backslash F$ is an element of $W$ and the pseudoline starting at level $i$ will end at level $w(i)$. We call one such arrangement a $w$-pseudoline arrangement. There is a one-to-one correspondence between facets $F$ of $\Delta(Q, w)$ and $\left(\prod Q \backslash F\right)$-pseudoline arrangements supported on $\mathcal{N}_{Q}$. In this setup, we have that $w(F, j)$ is the characteristic vector of the pseudolines passing below the $j$-th brick of $\mathcal{N}_{Q}$. Moreover, the $i$-th coordinate of the brick vector $B(F)$ is the number of bricks in $\mathcal{N}_{Q}$ that lie above the $i$-th pseudoline with contacts $F$, and the brick polytope $B(Q, w)$ is the following convex hull:

$$
B(Q, w):=\operatorname{conv}\left\{B(F): F \in \Delta(Q, w) \text { and } \prod Q \backslash F=w\right\}
$$

Example 3.5 Let $Q=\left(s_{1}, s_{2}, s_{1}, s_{2}, s_{1}\right)$ and $w=s_{1} s_{2} s_{1}$, then the pseudoline arrangement corresponding to the subword $J=\left(s_{1},-,-,-, s_{1}\right)$ gives the vector $B(J)=(2,0,2)$ obtained by counting bricks above each line. The brick polytope $B(Q, w)$ is pictured below.

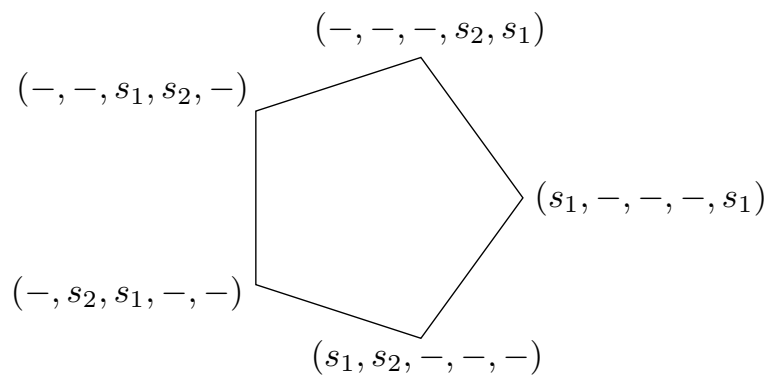

\subsection{Toric varieties for brick polytopes in the $S L_{n}(\mathbb{C})$ case}

We have already seen that subwords $R$ of $Q$ correspond to $T$-fixed points of $B S^{Q}$ and that if $p(R)$ is the point corresponding to $R$ then $m_{Q}(p(R))=\left(\prod Q \backslash R\right) B \in G / B$. This means that the rightmost flag of the point $p(R)$ is the flag corresponding to $\prod Q \backslash R \in W$ and so the pseudoline arrangement corresponding to $R$ is an $\left(\prod Q \backslash R\right)$-arrangement. The following example shows the correspondence.

Example 3.6 The pseudoline arrangement corresponding to the word $J=\left(s_{1},-,-,-, s_{1}\right)$ gives a $T$ fixed point of $B S^{\left(s_{1}, s_{2}, s_{1}, s_{2}, s_{1}\right)}$ as the following diagrams show 

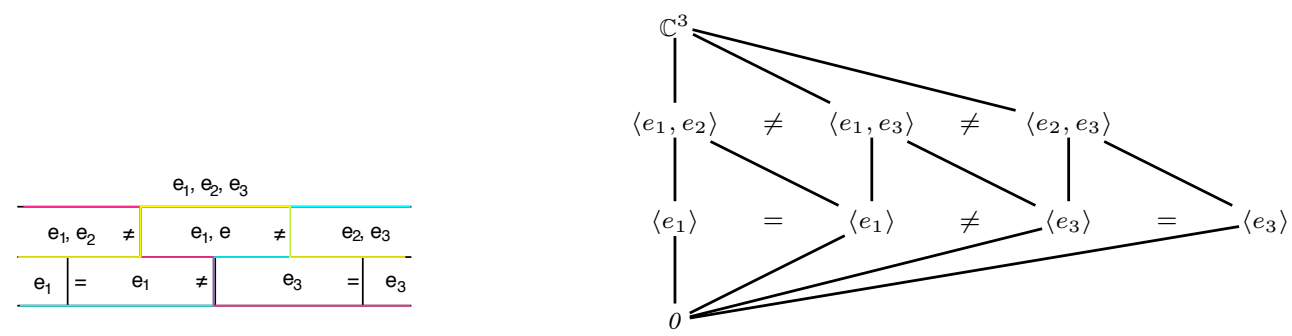

Theorem 3.7 Suppose $Q$ is toric and $w=\operatorname{Dem}(Q)$. There is a bijective correspondence between $w$ pseudoline arrangements supported by $\mathcal{N}_{Q}$ and T-fixed points of $m_{Q}^{-1}(w B)$ ). Moreover, this correspondence makes the moment map

$$
\mu: m_{Q}^{-1}(w B) \longrightarrow \mathbb{R}^{n}
$$

be equivalent to the mapping

$$
B:\left\{\text { w-pseudoline arrangements supported by } \mathcal{N}_{Q}\right\} \longrightarrow \mathbb{R}^{n}
$$

given in Pilaud and Santos (2012).

Proof: The first part of the proposition is proven in the paragraph preceding the example above. The second part of this theorem follows from using induction on $|Q|=m$ to prove that $\mu(I, j)=\mathrm{w}(I, j)$ for all subwords $I$ and all $j \in[m]$.

Theorem 3.8 The fiber $m_{Q}^{-1}(w B)$ is a toric variety if and only if $Q$ is toric, $\ell(w) \leq|Q| \leq \ell(w)+n$ and $\operatorname{Dem}(Q)=w$. Moreover, $m_{Q}^{-1}(w B)$ is the toric variety associated to the polytope $B(Q)$.

We have proved the if part of this theorem, however the only if part will be follow from Theorem 4.4 The following corollary follows from the work of Pilaud and Santos (2012). For the statement we define a Coxeter element $c$ to be the product of all simple reflections in some order. Define also the $c$-sorting word of $w$ to be the lexicographically first subword of $\mathbf{c}^{\infty}$ that is a reduced expression for $w$.

Corollary 3.9 If $Q$ is the word starting with a word $\mathrm{c}$ representing a Coxeter element $c$ and then having the c-sorting word for $w_{0}$, then $m_{Q}^{-1}\left(w_{0} B\right)$ is the toric variety of the associahedron as realized in Hohlweg and Lange (2007) and in Pilaud and Santos (2012).

Example 3.10 The toric variety of the pentagon from example 3.5 i.e. the associahedron corresponding to the Coxeter element $c=\left(s_{1}, s_{2}\right)$, is

$$
m_{Q}^{-1}(w B)=\left\{\left(V_{1}, V_{2}, V_{3}\right) \text { : each ascending chain in the poset below is a complete flag }\right\}
$$




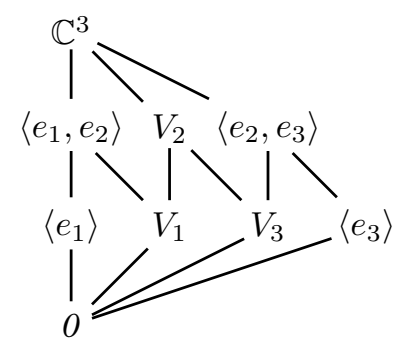

\section{Brick manifolds in the general case}

Let $G$ be a complex semisimple Lie group, let $B$ be a Borel subgroup of $G$, i.e., a maximal solvable subgroup, and $T$ be a maximal torus contained in $B$. Let $W$ be the Weyl group of $G$ with generators $S=\left\{s_{1}, \ldots, s_{n}\right\}$, which correspond to the simple roots $\Delta(W)=\left\{\alpha_{1}, \ldots, \alpha_{n}\right\}$. Let $P$ be a parabolic subgroup of $G$, i.e., a subgroup of $G$ for which the quotient $B / P$ is a projective algebraic variety; this condition is equivalent to $P$ contains $B$. We denote by $P_{i}$ the minimal parabolic subgroup corresponding to $s_{i}$, we then have that $P_{i} / B \cong \mathbb{C P}^{1}$. The torus $T$ acts on this quotient and this action has exactly two $T$-fixed points: one corresponding to the identity element and one corresponding to the generator $s_{i}$.

Definition 4.1 Let $Q=\left(s_{i_{1}}, \ldots, s_{i_{m}}\right)$ be a word in the generators of $W$. Then the product $P_{i_{1}} \times \cdots \times P_{i_{m}}$ has an action of $B^{m}$ given by:

$$
\left(b_{1}, \ldots, b_{m}\right) \cdot\left(p_{1}, \ldots, p_{m}\right)=\left(p_{1} b_{1}, b_{1}^{-1} p_{2} b_{2}, \ldots, b_{m-1}^{-1} p_{m} b_{m}\right)
$$

The Bott-Samelson variety of $Q$ is the quotient of the product of the $P_{i}$ 's by this action

$$
B S^{Q}:=\left(P_{i_{1}} \times \cdots \times P_{i_{m}}\right) / B^{m}
$$

Bott-Samelson varieties are smooth, irreducible and $|Q|$-dimensional algebraic varieties. These varieties come equipped with a natural map

$$
\begin{aligned}
B S^{Q} & \stackrel{\mathrm{m}_{\mathrm{Q}}}{\longmapsto} G / B \\
\left(p_{1}, \ldots, p_{m}\right) & \longmapsto\left(p_{1} \cdots p_{m}\right) B .
\end{aligned}
$$

The image of this map is the opposite Schubert cell $X^{w}:=\overline{B w B}$, where $w=\operatorname{Dem}(Q)$. In the case in which $Q$ is reduced, this map is a resolution of singularities for non-smooth $X^{w}$, however in this paper we will study cases in which $Q$ is not reduced.

Definition 4.2 Let $Q=\left(s_{i_{1}}, \ldots, s_{i_{m}}\right)$ be a word in the generators of $W$ and $w=\operatorname{Dem}(Q)$, then the Brick manifold is the fiber $m_{Q}^{-1}((B) w)$.

\subsection{Symplectic Structure on Bott-Samelson Varieties}

Let $P_{\hat{i}}$ be the maximal parabolic subgroup of $G$ corresponding to $S_{\hat{i}}:=\left\{s_{1}, \ldots, \hat{s}_{i}, \ldots, s_{n}\right\}$. Note that for $G=S L_{n}(\mathbb{C})$ each quotient $G / P_{\hat{i}}$ is a Grassmannian. Let $K$ be the maximal compact subgroup of $G$. Then we can view $G / P_{\hat{i}}$ as a coadjoint orbit, i.e., a $K$-orbit trough the fundamental weight $\lambda_{s_{i}} \in \mathfrak{k}^{*}$, 
where $\mathfrak{k}$ is the Lie algebra of $K$. This interpretation gives us a symplectic structure on $G / P_{\hat{i}}$ with respect to the action of $K$ such that the inclusion

$$
G / P_{\hat{i}} \longleftrightarrow \mathfrak{k}^{*}
$$

is a moment map for the $K$-action. Then the composition

$$
G / P_{\hat{i}} \longrightarrow \mathfrak{k}^{*} \longrightarrow \mathfrak{t}^{*}
$$

is the moment map of $G / P_{\hat{i}}$ with respect to the Torus action, where $t$ is the Lie algebra of the torus. Moreover, the moment map for the diagonal $T$-action on a product $\prod G / P_{\hat{i}}$ is the sum of each moment $\operatorname{map} G / P_{\hat{i}} \longrightarrow \mathfrak{t}^{*}$.

Let $T$ act on $B S^{Q}$ by

$$
t \cdot\left(p_{1}, p_{2}, \ldots, p_{m}\right)=\left(t \cdot p_{1}, p_{2}, \ldots, p_{m}\right) .
$$

Given $Q=\left(q_{1}, \ldots, q_{m}\right)$ we have a $T$-equivariant map

$$
B S^{Q} \stackrel{\varphi}{\longleftrightarrow} \prod_{i: s_{i} \in Q} G / P_{\hat{i}} .
$$

where $\varphi=\left(\varphi_{1}, \ldots, \varphi_{m}\right)$ and the $k$-th component is

$$
\begin{aligned}
B S^{Q} \stackrel{\varphi_{\mathrm{k}}}{\longrightarrow} G / P_{\hat{k}} \\
\left(p_{1}, \ldots, p_{m}\right) \longmapsto\left(\prod_{i<j} p_{i}\right) P_{\hat{k}} .
\end{aligned}
$$

The composition

$$
B S^{Q} \stackrel{\varphi}{\longleftrightarrow} \prod_{i: s_{i} \in Q} G / P_{\hat{i}} \longrightarrow \mathfrak{t}^{*}
$$

gives us a moment map for this Bott-Samelson variety with respect to the $T$-action. Thus Bott-Samelson varieties are Hamiltonian symplectic manifolds with respect to this torus action. The image of this map is the moment polytope, the convex hull of the images of the $T$-fixed points. This result is a theorem proven independently in Atiyah (1982) and in Guillemin and Sternberg (1982). There is a correspondence between $T$-fixed points on $B S^{Q}$ and subwords $R$ of $Q$ and if $p(R)$ is the $T$-fixed point corresponding to $S$ then

$$
m_{Q}(p(R))=\left(\prod Q \backslash R\right) B \in G / B
$$

This correspondence motivates the relation between fibers of the map $m_{Q}: B S^{Q} \longrightarrow G / B$ and subword complexes.

We now describe the image of the $T$-fixed points under the moment map. For each $k$ we have the moment map

$$
\mu_{k}: G / P_{\hat{k}} \longrightarrow \mathfrak{t}^{*}
$$

where $\mu_{k}\left(P_{\hat{k}}\right)=\lambda_{s_{k}}$, the fundamental weight corresponding to $s_{k}$, and it maps a general element to a Weyl conjugate of this fundamental weight. Before we finish describing the maps $\mu_{k}$, we note that the moment map of $B S^{Q}$ is then

$$
\sum_{k=1}^{m} \varphi_{k} \circ \mu_{k}
$$


Consider the fixed point $\left(p_{1}, \ldots, p_{m}\right)$ in $B S^{Q}$ corresponding to the subword $R$ of $Q$ then under the moment map $\mu_{k}$ each $p_{j}$ corresponds to either the reflection $s_{i_{j}}$ if $q_{j} \in S$ or to the identity in $W$. In other words, $p_{j}$ corresponds to $s_{i_{j}}$ if $p_{j} \notin B$ and to the identity in $W$ otherwise. In conclusion we have that for $R$ subword of $Q$ and

$$
\begin{gathered}
p_{R}=\text { the fixed point corresponding to } R \\
B S^{Q} \stackrel{\varphi_{\mathrm{k}} \circ \mu_{\mathrm{k}}}{\longrightarrow} \mathrm{t}^{*} \\
p_{R} \longmapsto\left(\prod_{i<k, q_{i} \in S} q_{i}\right)\left(\lambda_{s_{q_{k}}}\right) .
\end{gathered}
$$

It then follows that

$$
\begin{aligned}
B S^{Q} \stackrel{\mu}{\longrightarrow} \mathfrak{t}^{*} & \\
p_{R} & \longmapsto \sum_{k=1}^{m}\left(\prod_{i<k, q_{i} \in S} q_{i}\right)\left(\lambda_{s_{q_{k}}}\right)
\end{aligned}
$$

\subsection{Moment polytopes of Brick Manifolds}

We now state and prove the main results of the paper.

Theorem 4.3 The image of $m_{Q}^{-1}(B)$ under the moment map is the brick polytope $B(Q, w)$.

Proof: $T$-fixed points of $B S^{Q}$ are in 1-1 correspondence with subwords $R$ of $Q$. This induces a 1-1 correspondence between $T$-fixed points of $m_{Q}^{-1}$ (B) and the subwords $R$ of $Q$ with $\prod Q \backslash R=w$. If the subword $R$ is not a facet of the subword complex $\Delta(Q, w)$ then it gives a non reduced product $\prod Q \backslash R$. This implies that the root configuration $r(R)=\{\{r(R, i): i \in F\}\}$ has a smaller dimension than the root configuration of a facet and thus it cannot be a vertex. Therefore, moment polytope is the convex hull of the points corresponding to facets of $\Delta(Q, w)$ and by Equation 3 the image of each fixed point is precisely the one defined in Equation 1 in Section 2.2

Theorem 4.4 The fiber $m_{Q}^{-1}(w B)$ is a toric variety if and only if $Q$ is toric, $\ell(w) \leq|Q| \leq \ell(w)+$ $\operatorname{dim}(W)$ and $\operatorname{Dem}(Q)=w$. Moreover, $m_{Q}^{-1}(w B)$ is the toric variety associated to the polytope $B(Q, w)$.

Proof: We sketch the proof for the case $|Q|=\ell(w)+\operatorname{dim}(W)$. Note that $\operatorname{dim}(T)=\operatorname{dim}\left(m_{Q}^{-1}(w B)\right)$. It suffices to show that $T$ doesn't have generic stabilizer of positive dimension. This is true if and only if $\mu\left(m_{Q}^{-1}(w B)\right)$ spans $\mathbb{R}^{n}$. This happens precisely when $Q$ is toric.

\section{Acknowledgements}

I would like to thank my advisor Allen Knutson sharing this project with me and for all the incredibly fruitful meetings that happen at Cornell University. 


\section{References}

M. F. Atiyah. Convexity and commuting Hamiltonians. Bull. London Math. Soc., 14(1):1-15, 1982. ISSN 0024-6093. doi: 10.1112/blms/14.1.1. URL http://dx.doi.org/10.1112/blms/14.1.1.

R. Bott and H. Samelson. The cohomology ring of G/T. Proc. Nat. Acad. Sci. U. S. A., 41:490-493, 1955. ISSN 0027-8424.

R. Bott and H. Samelson. Correction to "Applications of the theory of Morse to symmetric spaces". Amer. J. Math., 83:207-208, 1961. ISSN 0002-9327.

C. Ceballos, J.-P. Labbé, and C. Stump. Subword complexes, cluster complexes, and generalized multiassociahedra. eprint arXiv:1108.1776v4, 2011. URL/http://arxiv.org/abs/arXiv:1108. $1776 \mathrm{v} 4$.

V. Guillemin and S. Sternberg. Convexity properties of the moment mapping. Invent. Math., 67(3):491513, 1982. ISSN 0020-9910. doi: 10.1007/BF01398933. URL http://dx.doi.org/10.1007/ BF 01398933 .

C. Hohlweg and C. E. M. C. Lange. Realizations of the associahedron and cyclohedron. Discrete Comput. Geom., 37(4):517-543, 2007. ISSN 0179-5376. doi: 10.1007/s00454-007-1319-6. URL http: //dx.doi.org/10.1007/s00454-007-1319-6.

A. Knutson. The symplectic and algebraic geometry of Horn's problem. Linear Algebra Appl., 319 (1-3):61-81, 2000. ISSN 0024-3795. doi: 10.1016/S0024-3795(00)00220-2. URL http://dx . doi.org/10.1016/S0024-3795(00)00220-2. Special Issue: Workshop on Geometric and Combinatorial Methods in the Hermitian Sum Spectral Problem (Coimbra, 1999).

A. Knutson and E. Miller. Subword complexes in Coxeter groups. Adv. Math., 184(1):161-176, 2004. ISSN 0001-8708. doi: 10.1016/S0001-8708(03)00142-7. URL http://dx.doi.org/10.1016/ S0001-8708(03)00142-7.

P. Magyar. Schubert polynomials and Bott-Samelson varieties. Comment. Math. Helv., 73(4):603-636, 1998. ISSN 0010-2571. doi: 10.1007/s000140050071. URL http://dx.doi.org/10.1007/ s000140050071.

V. Pilaud and M. Pocchiola. Multitriangulations, pseudotriangulations and primitive sorting networks. Discrete Comput. Geom., 48(1):142-191, 2012. ISSN 0179-5376. doi: 10.1007/s00454-012-9408-6. URL http://dx.doi.org/10.1007/s00454-012-9408-6

V. Pilaud and F. Santos. The brick polytope of a sorting network. European J. Combin., 33(4):632-662, 2012. ISSN 0195-6698. doi: 10.1016/j.ejc.2011.12.003. URL http://dx.doi.org/10.1016/ j.ejc.2011.12.003.

V. Pilaud and C. Stump. Brick polytopes of spherical subword complexes: A new approach to generalized associahedra. eprint arXiv:1111.3349, 2011. URL http://arxiv.org/abs/1111.3349.

N. Reading and D. E. Speyer. Cambrian fans. J. Eur. Math. Soc. (JEMS), 11(2):407-447, 2009. ISSN 1435-9855. doi: 10.4171/JEMS/155. URL http://dx.doi.org/10.4171/JEMS/155. 
C. Stump. A new perspective on $k$-triangulations. J. Combin. Theory Ser. A, 118(6):1794-1800, 2011. ISSN 0097-3165. doi: 10.1016/j.jcta.2011.03.001. URL http://dx.doi.org/10.1016/j. jeta.2011.03.001. 